\title{
Characterization of the trp5-27 allele used to monitor drug-induced mitotic gene conversion in the Saccharomyces cerevisiae tester strain D7
}

\section{Ann E.Ehrenhofer-Murray, Friedrich E.Würgler and Christian Sengstag 1}

\author{
Institute of Toxicology, Swiss Federal Institute of Technology and \\ University of Zürich, Schorenstrasse 16, CH-8603 Schwerzenbach, \\ Switzerland \\ ${ }^{1}$ To whom correspondence should be addressed
}

Mitotic gene conversions, among other recombinagenic events, can play an important role in the multistep process of carcinogenesis. The ability of chemicals to induce such gene conversions can easily be monitored in the Saccharomyces cerevisiae tester strain YHE2, a derivative of strain D7. For the detection of drug-induced gene conversions, two mutations in the TRP5 locus are used, tpp5-12 and trp5-27. Here we report on the characterization of the stable allele tpp5-27. Our analysis revealed two relevant mutations in trp5-27: (a) a transition $\mathrm{C}$ to $\mathrm{T}$ at position 121 after ATG that results in an amber stop codon and abolishes gene expression and (b) a transversion $A$ to $T$ at position 1555 that creates an ochre stop codon. Simultaneous amber and ochre suppression with the suppressors $S U P 3$ and $S U P 11$, respectively, was capable of relieving the tryptophan-requiring phenotype of strains carrying the top5-27 allele. These findings have implications on the length of gene conversion tracts in conversion events between trp5-12 and trp5-27: conversion tracts can cover several kilobases, if the site of the mutation in tpp-12 lies outside of the positions mutated in trp5-27. Conversely, the maximal length is limited to $1435 \mathrm{bp}$, if the mutation in trp5-12 is located between the positions mutated in trp5-27.

\section{Introduction}

Mitotic gene conversion is a process of nonreciprocal transfer of genetic information between homologous chromatid regions of chromosomes. In cells that are heterozygous for two alleles of a marker gene on the two homologous chromosomes, gene conversion within the region of the marker gene can result in the loss of heterozygosity, and a clone can develop that is homozygous for one of the marker alleles (Würgler, 1992). If in proliferating tissue a cell turns homozygous for a pre-existing mutation in a tumor suppressor gene that leads to the loss of gene function, this loss of heterozygosity will enable the phenotypical manifestation of the mutation and may result in increased proliferation of the cell (Sengstag, 1994). Thereby, gene conversions, among other recombinagenic events, represent a possible step in the multistep process of carcinogenesis (Fearon and Vogelstein, 1990). Chemical compounds that raise gene conversion frequencies are, therefore, thought to contribute considerably to the development of neoplasms. In addition to their effect on cancer induction, promotion and progression, gene conversion events leading to loss of heterozygosity can also affect other biological functions that influence health (Würgler, 1992). During the last few years, the awareness of the importance of such processes has substantially increased, and various test systems have been designed to identify the recombinational properties of substances which might be indicative of their carcinogenic potential (Sengstag, 1994). One of these test systems, the Saccharomyces cerevisiae tester strain D7 (Zimmermann et al., 1975), allows the detection of gene conversions simultaneously with reversions and mitotic recombinations. As a unicellular eukaryote, S.cerevisiae provides a readily manipulable, yet genetically complex model organism suitable for the study of recombinational events (Zimmermann, 1992), and since its introduction the tester strain D7 has been extensively used for genotoxicity testing (Zimmermann et al., 1984). As the yeast cells lack certain enzymatic activities that can be crucial for the activation of procarcinogens, various human enzymes have been heterologously expressed in a yeast strain derived from D7 (Eugster et al., 1990, 1991, 1992) and are capable of conferring such metabolic competence to yeast (Eugster et al., 1993; Eugster and Sengstag, 1993; Sengstag et al., 1994). This approach has proved promising in broadening the applicability of the test system.

The process of gene conversion can easily be followed in heteroallelic diploid yeast strains that carry two defective alleles of the same gene locus (Zimmermann, 1975). In strain D7 (Zimmermann et al., 1975), two non-complementing alleles of the tryptophan synthase gene TRP5 (Zalkin and Yanofsky, 1982) are combined. Taken on their own, the two alleles trp5-12 and trp5-27 are completely stable (trp5-27) or exert low reversion frequencies (trp5-12, reversion frequency $10^{-6}$ ) that do not interfere with gene conversion (Zimmermann and Schwaier, 1967). Conversely, the tryptophan-requiring heteroallelic diploid D7 generates tryptophan nonrequiring convertants at a frequency of approximately $10^{-5}$. The conversion frequency can be increased by treatment of the cells with DNA-damaging agents (Zimmermann et al., 1975). For our genotoxicity testing, we are working with a ura3 derivative of D7, YHE2 (Eugster et al., 1990). We were interested to characterize the trp5 mutations present in our tester strain in order to gain more insight into the gene conversion events taking place between the two trp 5 alleles. Here we report the characterization of one of the alleles, trp5-27.

\section{Materials and methods \\ Yeast and bacterial strains, media and culture conditions}

The construction of the S.cerevisiae strains YHE2 ( $\mathrm{a} / \alpha$, ade2-40/ade2-119, ilv1-92/ilv1-92, trp5-27/trp5-12, ura3 $\Delta 5 /$ ra $3 \Delta 5$ ), and YHE1 ( $\alpha$, ade2-40, ilv1-92, trp5-27, ura3 $\Delta 5$ ), has previously been described (Eugster et al., 1990). Strain YAE76 (a, ade 2-119, itv1-92, trp5-111, ura3 $\Delta 5$ ) was constructed in this laboratory. Strain JRY1631 ( $\alpha$, ade2-110, cyh2, his 3 $\Delta 200$, leul, hys2-801, trp5, ura3-52) was kindly provided by Di J.Rine. Yeast transformations were performed as described by Klebe et al. (1983). The bacterial strain $\mathrm{DHS \alpha F}^{\prime}$ was used for propagation of recombinant plasmids.

Yeast strains were grown in standard media. Minimal medium YM [0.67\% yeast nitrogen base without amino acids (Difco), $2 \%$ glucose] was appropriately supplemented with amino acids as described by Sherman (1991). Escherichia coli was grown in LB medium which was supplemented with $150 \mu \mathrm{g} / \mathrm{ml}$ ampicillin where appropriate.

Plasmid constructions

Plasmid pAE158 is a derivative of YEp352 (Hill et al., 1986) and contains in its BamHI site the TRP5 gene from pYAS1 (Dohmen et al., 1989) as a $3.3 \mathrm{~kb}$ BamHI fragment. In PAE158, TRP5 has the same orientation as lacZ. Plasmid pAE268 is analogous to pAE158, but contains the trp5-27 gene which was cloned 
from YHE1 (see Results). Plasmids pAE470, pAE471, pAE475 and pAE491 are derived from pAE268 and have their BglII/Sall, Spel/SalI, NcoI anc Bst EII/Sal I trp5-27 fragments, respectively, replaced by the corresponding TRP5 sequence. Plasmids pRS313SUP3, pRS316SUP3 and pUN60SUP3 were constructed by introducing the $137 \mathrm{bp}$ SUP 3 BamHI fragment of mWJ64 (Guc and Wu, 1982) into the BamHI sites of pRS313, pRS316 (Sikorski and Hieter 1989) and pUN60 (Elledge and Davis, 1988), respectively. To obtain pAE492, the blunt-ended trp5-27 BamHI fragment of pAE268 was introduced into the $\mathrm{XbaL} / \mathrm{Nat} \mathrm{I}$ cleaved, blunt-ended plasmid pRS316SUP3 to give pAE342. Subsequently, the PruII fragment of pAE342 containing trp5-27 and SUP3 was cloned in the PuII cut vector pRS313. Plasmid pAE496 was constructed by cloning the Saci/SalI trp5-27 fragment of pAE268 in the Sact/SalI cleaved vector pRS313.

\section{Colony hybridization and derection}

Bacterial colonies representing the BamHI/HindIII sublibrary of YHE1 in pRS316 was transferred to Pall Biodyne A transfer membranes, lysed and their DNA bound to the filters as described by Sambrook et al. (1989). For the detection of TRPS-containing colonies, the $3.3 \mathrm{~kb}$ BamHI fragment of pAE158 was digoxygenin-labelled with the DIG DNA Labeling and Detection Kit from Bochringer. Labelling, hybridization and chemiluminescent detection were performed according to the supplier's protocols.

DNA sequence analysis

Fragments to be sequenced were subcloned in pBLKS and pBLSK (Stratagene). DNA sequence analysis was performed on single-stranded DNA by the dideoxy chain termination method (Sanger et al., 1977) using the Sequenase Version 2.0 DNA Sequencing Kit (US Biochemical Corporation) sccording to the manufacturer's specification. $\left[\alpha-{ }^{35} S\right]$ dATP was purchased from DuPont.

\section{Results}

\section{Cloning of the trp5-27 gene}

In order to clone the trp5-27 gene, a genomic sublibrary of the S.cerevisiae trp5-27 strain YHE1 was constructed in the E.coli/ S.cerevisiae shuttle vector pRS316. To this end, total DNA isolated from YHEl was double-digested with BamHI and HindIII, which divides the trp 5-27 gene in two fragments of 1815 and $1516 \mathrm{bp}$ length. DNA fragments in a size range of $1.4-1.9 \mathrm{~kb}$ were purified from an agarose gel and ligated to the BamHI and HindIII cleaved vector pRS316. Four hundred and fifty individual ampicillin resistant $E$.coli transformants were used for a colony hybridization experiment (see Materials and methods). As a probe, the $3.3 \mathrm{~kb}$ digoxigenin-labelled TRP5 BamHI fragment of pAE158 was taken. To confirm the identity of the 11 clones that gave rise to positive signals, their plasmid DNA was subjected to restriction analysis. By this procedure, two clones representing the 1.8 and $1.5 \mathrm{~kb}$ fragments of the $\operatorname{trp5}$-27 were chosen for further characterization. Subsequently, the complete trp5-27 gene was reconstituted in the E.coli/S. cerevisiae shuttle vector YEp352 by triple-ligating the two individual trp5-27 BamHI/HindIII fragments to the BamHI cleaved YEp352 to give pAE268.

The phenotype conferred by the trp5-27 plasmid pAE268 was determined by transforming strain YHE1 to uracil prototrophy with pAE268 and, as a control, with pAE158, which is a YEp352 derivative and carries the wild-type TRP5 gene. YHE1 pAE268 and YHE1 pAE158 were streaked on supplemented minimal medium lacking uracil or uracil and tryptophan, and the plates were incubated for 3 days at $30^{\circ} \mathrm{C}$ (Figure 1A). In contrast to YHE1 transformed with the TRP5-carrying plasmid, YHE1 containing the trp5-27 plasmid was unable to grow on medium lacking tryptophan. Thus, plasmid pAE268 does not confer tryptophan prototrophy to $\mathrm{YHEl}$ and, therefore, presumably contains a mutation in its trp5-27 allele that distinguishes it from pAE158.

\section{Mapping and sequencing of an amber mutation in trp5-27}

Since knowledge of the underlying mutation(s) in the trp5-27 and trp5-12 alleles present in the S.cerevisiae strains D7 and YHE2 would give us a more detailed insight into the gene conversion

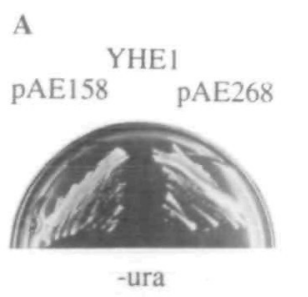

B

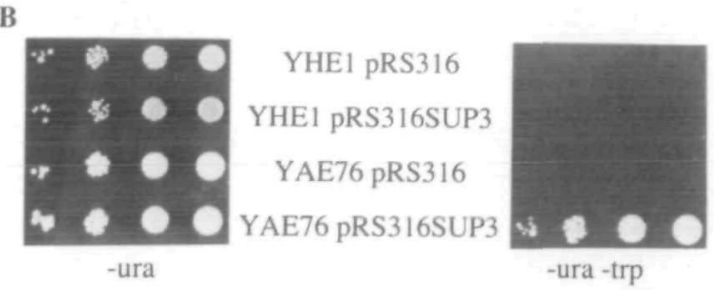

Fig. 1. (A) Comparison of the tryptophan phenotypes conferred to YHE1 by plasmids carrying TRP5 (pAE158) and trp5-27 (pAE268). YHE1 pAE158 and YHE1 pAE268 were streaked on supplemented minimal medium lacking uracil (-ura) or uracil and tryptophan (-ura - trp) and incubated for 3 days at $30^{\circ} \mathrm{C}$. (B) Amber suppression in YHE1 (trp5-27) and YAE76 (trp5-111). The strains transformed with a vector control (pRS316) or an amber suppressor containing plasmid (pRS316SUP3) were pregrown in supplemented minimal medium to stationary phase and diluted to an absorbance at $600 \mathrm{~nm}$ of $0.3 .5 \mu \mathrm{l}$ of these cell suspensions and of $1: 10$, $1: 100$ and 1:1000 dilutions were placed on supplemented minimal medium lacking uracil (-ura) or uracil and tryptophan (-ura - trp). Plates were incubated for 3 days at $30^{\circ} \mathrm{C}$.

\section{Trp suppression phenotype with SUP3}
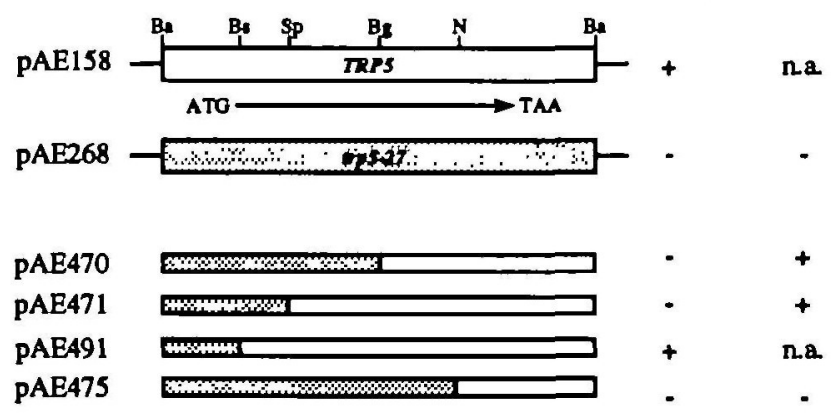

Fig. 2. Replacement of plasmid-bome tmp5-27 segments by wild-type TRPS and determination of the phenotype conferred by these plasmids. The white and grey boxes represent the TRP5 and trp5-27 sequences, respectively. The phenotypes conferred by the plasmids were determined in strain YHE1 and are indicated as $(+)$ for tryptophan prototrophy and (-) for tryptophan auxotrophy. By testing the tryptophan phenotype of JRY 1631 couransformed with respective $t r p 5$ plasmids and pRS313SUP3, novel trp5 alleles derived from trp5-27 were identified whose tryptophan conferring phenotype was $(+)$ or was not (-) amber suppressible (n.a., not applicable). The diroction and approximate length of the TRPS open reading frame are indicated by the arrow. Selected restriction sites are indicated $(\mathrm{Ba}=\mathrm{Bam} \mathrm{H}$, $\mathrm{Bg}=\mathrm{Bg} l \mathrm{II}, \mathrm{Bs}=B_{s t} \mathrm{EII}, \mathrm{N}=\mathrm{NcoI}, \mathrm{Sp}=$ Spel).

events, we were interested in localizing the site of the mutation(s) in the trp5-27 allele. Therefore, we replaced fragments of increasing length of the trp5-27 plasmid pAE268 by the corresponding TRP5 fragment and tested the tryptophan phenotype conferred by the new constructs (Figure 2). For this purpose, YHE1 was transformed with each novel construct and Ura ${ }^{+}$ transformants were streaked on supplemented minimal medium lacking uracil or tryptophan and uracil. A tryptophan nonrequiring phenotype was only observed when $\operatorname{trp5-27}$ sequences downstream of the Bst EII site were replaced by the corresponding wild-type sequences, while replacement of the sequences downstream of the SpeI site was not sufficient to confer tryptophan 
JRY 1631

$\begin{array}{ll}\text { pRS316 } & \text { pRS313SUP3 } \\ \text { pAE158 } & \text { pRS313 } \\ \text { pAE268 } & \text { pRS313SUP3 } \\ \text { pAE470 } & \text { pRS313 } \\ \text { pAE470 } & \text { pRS313SUP3 }\end{array}$
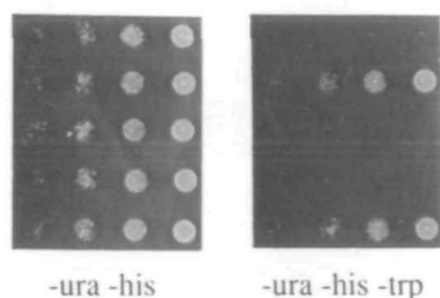

Fig. 3. Amber suppression of various trp5 alleles in JRY1631. JRY1631 cotransformed with the indicated plasmids was grown to stationary phase in liquid medium and diluted to $3 \times 10^{6} \mathrm{cells} / \mathrm{ml} ; 5 \mu \mathrm{l}$ of these and of $1: 10$, 1:100 and 1:1000 dilutions were spotted on supplemented minimal medium lacking uracil and histidine (-ura - his) or uracil, histidine and tryptophan (-ura - his - trp). The plates were incubated for 2 days at $30^{\circ} \mathrm{C}$.

prototrophy. This allowed us to map the site(s) of the mutation(s) in $t r p 5-27$ to a $380 \mathrm{bp} \mathrm{BstEII/SpeI} \mathrm{fragment.} \mathrm{To} \mathrm{elucidate} \mathrm{the}$ exact nature of the mutation(s) assumed to lie in this DNA segment, the DNA sequence of the corresponding trp5-27 fragment was determined. The fragment was cloned into pBLKS and pBLSK, single-stranded DNA was prepared and sequenced according to the dideoxy method. Comparison of the trp5-27 sequence with the wild-type TRP5 sequence revealed a transition $\mathrm{C}$ to $\mathrm{T}$ at nucleotide position 121 after ATG. This mutation results in an amber stop codon TAG in trp5-27 and represents a potential cause for the Trp ${ }^{-}$phenotype observed for the trp5-27 allele.

\section{Attempted suppression of the trp5-27 amber mutation}

To determine whether the amber mutation found on trp5-27 plasmid pAE268 was the only mutation responsible for the tryptophan auxotrophic phenotype conferred by the plasmid, we attempted to suppress this Trp ${ }^{-}$phenotype with the amber suppressor SUP3. In order to achieve this objective, S.cerevisiae strain JRY1631 with the relevant genotype trp5, his $3 \Delta 200$, ura3-52 was cotransformed with either YEp352 or pAE268 as URA3 plasmids and either pRS313 or pRS313SUP3 as HIS3 plasmids. Ura ${ }^{+} / \mathrm{His}^{+}$transformants were grown in liquid culture to stationary phase. Aliquots of serial dilutions were spotted on supplemented minimal medium lacking uracil and histidine or tryptophan, uracil and histidine, and the plates were incubated at $30^{\circ} \mathrm{C}$ for 2 days (Figure 3). The trp5 mutation of JRY1631 was not suppressible with SUP3 as shown by the inability of JRY1631 pRS316 pRS313SUP3 to grow without tryptophan. Surprisingly, JRY1631 carrying PAE268 and the SUP3-containing plasmid was also unable to grow without tryptophan, indicating that the $\operatorname{Trp}^{-}$phenotype conferred by pAE68 was not amber-suppressible.

To make sure that the inability to amber-suppress JRY 1631 pAE268 was not due to a cloning artefact on pAE268, but that the cloned $t r p 5-27$ allele was identical with the allele present in YHE1, we attempted to suppress the tryptophan auxotrophic phenotype of YHEl with the amber suppressor SUP3. YHEI was transformed with the control vector pRS316 and with pRS316SUP3. In parallel, strain YAE76, which has an amber-suppressible tryptophan-requiring phenotype (A.Ehrenhofer-Murray and C.Sengstag, unpublished), was transformed with the same plasmids in order to confirm functional expression of SUP3 in pRS316SUP3. Ura ${ }^{+}$transformants were grown in liquid culture to stationary phase and diluted to $3 \times 10^{6}$ cells $/ \mathrm{ml}$. Aliquots of serial dilutions were spotted on supplemented minimal medium lacking uracil or tryptophan and uracil, and the plates were incubated at $30^{\circ} \mathrm{C}$ for 3 days (Figure 1B). Only the amber suppression control strain YAE76 containing the SUP 3 plasmid could grow without tryptophan, while trp5-27 strain YHE1 containing SUP3 was unable to grow. Therefore, as found for the $t r p 5-27$ allele on PAE268, the $t r p 5-27$ mutation of YHE1 is not suppressible with the amber suppressor SUP3. These results suggested either that $S U P 3$ was not the appropriate amber suppressor for $\operatorname{trp} 5-27$, or that there was a second mutation present in $\operatorname{trp5-27}$ that restricted its amber suppressibility.

Amber suppression of novel trp5 alleles and identification of a second mutation in trp5-27

To rule out the possibility that the inability of SUP 3 to suppress the $\operatorname{Trp}^{-}$phenotype of $t r p 5-27$ was due to the nature of this suppressor, we attempted to amber-suppress novel trp5 alleles derived from trp5-27. This was achieved by exchanging fragments of pAE268 for the corresponding wild-type TRP5 fragment, cotransforming JRY1631 with the new constructs and either pRS313 or pRS313SUP3 to uracil and histidine prototrophy and determining the tryptophan phenotype of the transformants by streaking them on medium lacking uracil and histidine or tryptophan, uracil and histidine (Figure 2; c.f. also Figure 3). A trp 5 allele present on pAE470 with the sequence downstream of the $B g l \Pi$ site replaced by wild-type sequences was suppressible with SUP3, while substitution of the trp5-27 sequences downstream of the $\mathrm{NcoI}$ site conferred no suppressible phenotype. These results demonstrated that the suppressor SUP3 was in fact capable of suppressing certain trp5 alleles derived from trp 5-27. In addition, these results strongly suggested the presence of a further mutation within a $606 \mathrm{bp} \mathrm{Bgl \amalg INcoI} \mathrm{fragment} \mathrm{of} \mathrm{trp5-27}$ that interferes with amber suppression. In order to identify this potential second mutation, the trp5-27 Bgl $\mathrm{I} / \mathrm{Ncol}$ fragment was subjected to DNA sequence analysis. This revealed a transversion A to $\mathrm{T}$ at nucleotide position 1555 after ATG. This mutation creates an ochre stop codon TAA in trp5-27 that presumably was responsible for the inability to amber-suppress trp5-27.

\section{Simultaneous amber and ochre suppression of trp5-27}

To confirm that the amber and ochre mutations of trp5-27 at positions 121 and 1555 after ATG were the only mutations responsible for its tryptophan-requiring phenotype, we undertook experiments to suppress both mutations simultaneously. To this end, the HIS3 plasmids pAE496 and pAE492 were constructed that contain the trp 5-27 gene from YHE1 alone or with the amber suppressor SUP3, respectively. JRY1631 was cotransformed with pAE496 or pAE492 as HIS3 plasmids and pRS316 or pUN60 as URA3 plasmids. Plasmid pUN60 carries the ochre suppressor SUP11 (Elledge and Davis, 1988). Histidine and uracil prototrophic transformants were subsequently streaked on supplemented minimal medium lacking histidine and uracil or tryptophan, histidine and uracil, and the plates were incubated for 3 days at $30^{\circ} \mathrm{C}$ (Figure 4). The $\operatorname{Trp}^{-}$phenotype of JRY1631 was not suppressed with SUP3 or SUP1I alone or in combination (data not shown). Similarly, a JRY1631 transformant containing the trp5-27 plasmid and either SUP3 or SUP11 was unable to grow without tryptophan, whereas a transformant expressing trp5-27 and both SUP3 and SUP11 showed a tryptophan prototrophic phenotype. This result clearly demonstrated that simultaneous amber and ochre suppression was necessary and sufficient to cure the Trp ${ }^{-}$phenotype of $t r p 5-27$. Similarly, the Trp ${ }^{-}$phenotype of YHEl was suppressed by a plasmid containing both suppressors SUP3 and SUP11 (data not shown). 


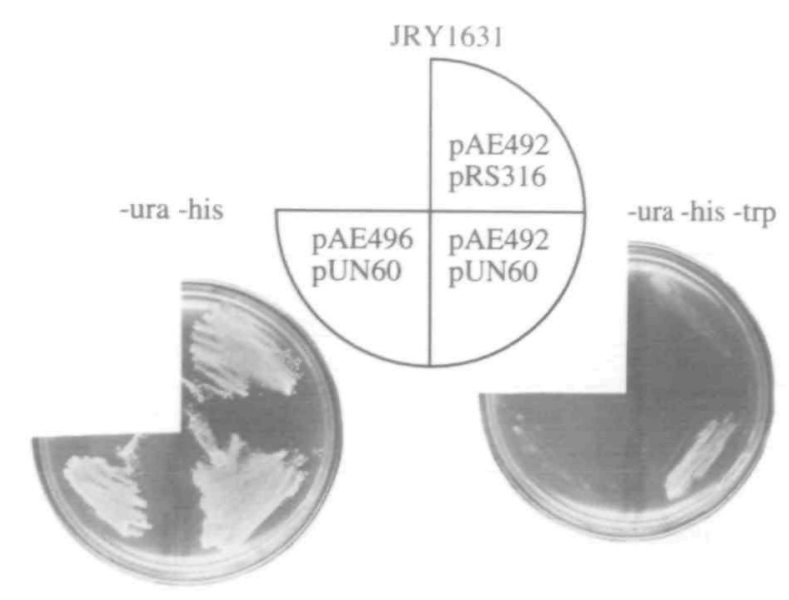

Fig. 4. Simultaneous amber and ochre suppression of trp5-27. JRY1631 transformants were streaked on supplemented minimal medium lacking uracil and histidine (- ura - his) or uracil, histidine and tryptophan (- ura - his -trp). The plates were incubated at $30^{\circ} \mathrm{C}$ for 3 days.

\section{Discussion}

The trp5-27 allele, one of the two defective alleles of the TRP5 locus (Zalkin and Yanofsky, 1982) in the diploid S.cerevisiae genotoxicity strain D7 (Zimmermann et al., 1975), has found extensive use in the detection and quantification of drug-induced gene conversion events. Despite its broad application, the mutation in trp5-27 so far has not been characterized and, thus, little is known about the nature of gene conversion events in D7 that produce tryptophan prototrophic convertants.

In this study, we have identified two relevant mutations in trp5-27 that are responsible for its stable tryptophan auxotrophic phenotype. At nucleotide position 121 after ATG, trp5-27 contained a transition $\mathrm{C}$ to $\mathrm{T}$ that results in an amber stop codon. This stop codon presumably terminates translation, giving rise to a truncated and probably abortive Trp5 gene product. The fact that suppression of this mutation with the amber suppressor SUP3 (Guo and Wu, 1982) did not suffice to cure the Trp ${ }^{-}$phenotype was not due to inherent characteristics of SUP3, since other, novel $\operatorname{trp} 5$ alleles derived from trp5-27 were in fact suppressible with SUP3. It rather indicated the presence of a second mutation in the trp5-27 gene. It was subsequently detected as a transversion A to T at nucleotide position 1555 after ATG and creates an ochre stop codon. Ochre suppression alone with SUP11 (Elledge and Davis, 1988) in trp5-27 did not result in tryptophan prototrophy. Conversely, simultaneous amber and ochre suppression was capable of relieving the tryptophan requirement of strains carrying trp5-27. Therefore, the two stop codons were the mutations relevant for the $\operatorname{Trp}^{-}$phenotype of $t r p 5-27$.

This finding has implications concerning the length of gene conversion tracts in conversion events between trp5-27 and trp5-12 that are successful in forming $\operatorname{Trp}^{+}$convertants in D7. In S.cerevisiae, gene conversion tracts are usually continuous, information being transferred as a single block from one chromosome to another (Borts and Haber, 1989). If transfer is to be directed from trp5-12 to trp5-27 (Figure 5A), a contiguous DNA stretch has to cover both mutations of trp5-27 and thus requires a minimal length of $1435 \mathrm{bp}$. Consequently, the assumed mutation in trp5-12 would lie outside of the positions mutated in $\operatorname{trp5-27}$, and therefore would be located either upstream of position 121 or downstream of position 1555 , presumably within the TRP5 open reading frame that ends at position 2619. In such a case, the conversion tract could expand over several kilobases
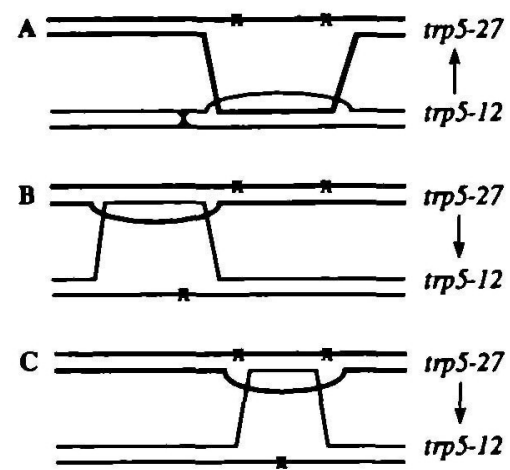

Fig. 5. Schematic representation of possible gene conversion events between trp5-27 and trp5-12 that result in $\mathrm{Trp}^{+}$convertants in $\mathrm{D} 7$. The approximate site of the relevant mutations in trp5-27 and one possible location of a mutation in trp5-12 are designated by crosses $(\mathrm{X})$. The directions of information transfer during gene conversion are indicated by arrows. Conversion tracts can cover several kilobases, if the site of the mutation in $t r p 5-12$ lies outside of the positions mutated in $t p 5$-27 (A and B). Conversely, the maximal tract length is limited to $1435 \mathrm{bp}$, if the mutation in $\operatorname{trp5}-12$ is located between the positions mutated in trp5-27 (C).

downstream or upstream of the trp5-12 mutation, respectively. Likewise, if information transfer proceeds from trp5-27 to a trp5-12 allele whose mutant site lies outside of the positions mutated in trp5-27, the conversion tract could also span several kilobases (Figure 5B). Conversely, its size would be limited to a maximum of $1435 \mathrm{bp}$, if the mutation in trp5-12 was located between the positions mutated in trp5-27 (Figure 5C). Judd and Petes (1988) measured the tract length of mitotic conversions of the ura3-3 allele at the URA3 locus and reported a minimum average conversion tract length of $0.9 \pm 0.8 \mathrm{~kb}$. In $40 \%$ of the cases, though, the average tract length was between 4 and $10 \mathrm{~kb}$. We therefore favour the model of the mutated site in $t$ p $5-12$ lying upstream of position 121 or downstream of position 1555 after ATG (Figure 5A and B), also since the conversion frequency of D7 $\left(10^{-5}\right.$; Zimmermann et al., 1975) compares well to the frequency in the ura3-3/URA3 strain $\left(4 \times 10^{-6}\right.$; Judd and Petes, 1988).

In light of these results, the characterization of the trp5-12 allele of D7 represents an important task and will give more precise information on the direction and extent of information transfer during gene conversion between the two alleles.

\section{Acknowledgements}

We wish to thank Drs H.P.Eugster, P.Niederberger, J.Rine and J.Thorner for generously sharing yeast strains and plasmids. This study was supported by a grant from the Swiss Cancer League to A.E.

\section{References}

Borts,R.H. and Haber,J.E. (1989) Length and distribution of meiotic gene conversion tracts and crossovers in Saccharomyces cerevisiae. Genetics, 123, $69-80$.

Dohmen,R.J., Strasser,A.W., Zitomer,R.S. and Hollenberg,C.P. (1989) Regulated overproduction of alpha-amylase by transformation of the amylolytic yeast Schwanniomyces occidentalis. Curr. Genet., 15, 319-325.

Elledge,S.J. and Davis,R.W. (1988) A family of versatile centromeric vectors designed for use in the sectoring-shuffle mutagenesis assay in Saccharomyces cerevisiae. Gene, 70, 303-312.

Eugster,H.P., Bartsch,S., Würgler,F.E. and Sengstag,C. (1992) Functionsl co-expression of human oxidoreductase and cytochrome P450 1Al in Sachianamyces cerevisiae results in increased EROD activity. Biochem. Biophys. Res. Commun., 185, 641-647.

Eugster,H.P., Probst,M., Würgler,F.E. and Sengstag,C. (1993) Caffeine, estradiol, and progesterone interact with human CYP1A1 and CYP1A2. 
Evidence from cDNA-directed expression in Saccharomyces cerevisiae. Drug Metab. Dispos. Biol. Fate Chem., 21, 43-49.

Eugster,H.P. and Sengstag,C. (1993) Saccharomyces cerevisiae: an alternative source for human microsomal liver enzymes and its use in drug interaction studies. Taricology, 82, 61-73.

Eugster,H.P., Sengstag,C., Hinnen,A., Meyer,U.A. and Würgler,F.E. (1991) Heterologous expression of human microsomal epoxide bydrolase in Sacchanamyces cerevisiae. Study of the valpromide-carbamazepine epoxide interaction. Biochem. Pharmacol, 42, 1367-1372.

Eugster,H.P., Sengstag,C., Meyer,U.A., Hinnen,A. and Würgler,F.E. (1990) Constitutive and inducible expression of human cytochrome P450IAl in yeast Saccharamyces cerevisiae: an alternative enzyme source for in vitro studies. Biochem. Biophys. Res. Commun., 172, 737-744.

Fearon,E.R. and Vogelstein,B. (1990) A genetic model for colorectal numorigenesis. Cell, 61, 759-767.

Guo,L.H. and Wu,R. (1982) New rapid methods for DNA sequencing based in exonuclease III digestion followed by repair synthesis. Nucleic Acids Res. $10,2065-2084$.

Hill,J.E., Meyers,A.M., Koerner,T.J. and Tzagoloff,A. (1986) Yeast/E.coli shuttle vectors with multiple unique restriction sites. Yeast, 2, 163-167.

Judd,S. and Petes, T. (1988) Physical lengths of meiotic and mitotic gene conversion tracts in Saccharomyces cerevisiae. Genetics, 118, 401-410.

Klebe,R.J., Harriss,J.V., Sharp,Z.D. and Douglas,M.G. (1983) A general method for polyethylene-glycol-induced genetic transformation of bacteria and yeast. Gene, 25, 333-341.

Sambrook,J., Fritsch,E.F. and Maniatis,T. (1989) Molecular Cloning; a Laboratory Manual, 2nd edn. Cold Spring Harbor Laboratory Press, Cold Spring Harbor, NY.

Sanger,F., Nicklen,S. and Coulson,A.R. (1977) DNA sequencing with chainterminating inhibitors. Proc. Natl. Acad. Sci. USA, 74, 5463-5467.

Sengstag,C., Eugster,H.P. and Würgler,F.E. (1994) High promutagen activating capacity of yeast microsomes containing human cytochrome P-450 1A and human NADPH-cytochrome P-450 reductase. Carcinogenesis, 15, 837-843.

Sengstag, C. (1994) The role of mitotic recombination in carcinogenesis. Crit. Rev. Taxicol., in press.

Sherman,F. (1991) Geating stared with yeast. Methods Erzymol., 194, 3-21.

Sikorski,R.S. and Hieter,P. (1989) A system of shuttle vectors and yeast host strains designed for efficient manipulation of DNA in Saccharamyces cerevisiae. Genetics, 122, 19-27.

Würgler,F.E. (1992) International Commission for Protection Against Environmental Mutagens and Carcinogens. Recombination and gene conversion. Mutat. Res., 284, 3-14.

Zalkin,H. and Yanofsky,C. (1982) Yeast gene TRP5: structure, function, regulation. J. Biol. Chem, 257, 1491-1500.

Zimmermann,F.K. (1975) Procedures used in the induction of mitotic recombination and mutation in the yeast Sacchanonyces cerevisiae. Mutar. Res. $31,71-86$

Zimmermann,F.K. (1992) Tests for recombinagens in fungi. Mutat. Res., 284, $147-158$.

Zimmermann,F.K., Kern,R. and Rasenberger,H. (1975) A yeast strain for simultaneous detection of induced mitotic crossing over, mitotic gene conversion and reverse mutation. Mutat. Res., 28, 381-388.

Zimmermann,F.K. and Schwaier,R. (1967) Induction and mitotic gene conversion with nitrous acid, 1-methyl-3-nitroguanidine and other alkylating agents in Saccharamyces cerevisiae. Mol. Gen. Genet., 100, 63-76.

Zimmermann,F.K., von Borstel,R.C., von Halle,E.S., Parry,J.M., Siebert,D., Zetterberg,G., Barale,R. and Loprieno,N. (1984) Testing of chemicals for genetic activity with Saccharomyces cerevisiae: a report of the U.S. Environmental Protection Agency Gene-Tox Program. Mutar. Res., 133, $199-244$

Received on February 14, 1994; accepted on April 11, 1994 